\title{
Antígeno carcinoembrionario elevado con estudio digestivo normal, ¿qué hacer?
}

\author{
Carcinoembryonic antigen raised with digestive \\ normal study, what to do?
}

\author{
Gargantilla $\mathrm{P}^{1,2}$, Arroyo $\mathrm{N}^{1}$, Pintor $\mathrm{E}^{2,}$ Montero $\mathrm{J}^{1}$ \\ ${ }^{1}$ Servicio de Medicina Interna. Hospital de El Escorial de Madrid. ${ }^{2}$ Universidad Europea de Madrid
}

\begin{abstract}
Resumen
El antigeno carcinoembrionario (CEA) es una glucoproteína intracelular que es producida por casi el 90\% de los cánceres colorectales y que puede ser medida cuantitativamente en suero. Esta sustancia también puede elevarse en otras situaciones como son los tumores gástricos, pancreáticos, pulmonares, de mama y en la neoplasia medular de tiroides, así como en enfermedades no neoplásicas como puede ser la cirrosis, la colitis ulcerosa y la pancreatitis.
\end{abstract}

Palabras clave: Antígeno carcinoembrionario, CEA. Cancer colorectal, Neoplasia medular de tiroides,

\section{Introducción}

El antígeno carcinoembrionario (CEA) fue descrito por vez primera por Gold y Freeman en $1965^{1}$ y es uno de los marcadores tumorales más empleados en la práctica clínica; en los pacientes que lo tienen elevado se debe realizar una investigación exhaustiva del aparato digestivo, encaminada a descartar procesos tumorales colónicos y gástricos mediante endoscopias, a pesar de que el paciente se encuentre asintomático. Presentamos el caso de una paciente asintomática que en una analítica rutinaria se detecta una elevación del CEA y en la que el estudio digestivo es normal.

\section{Caso clínico}

Se trata de una mujer de 38 años, sin antecedentes personales de interés y sin tratamiento en ese momento. Su médico de Atención Primaria realiza una analítica rutinaria en la que destaca como único hallazgo CEA $200 \mathrm{ng} / \mathrm{ml}$ (valor normal $<5 \mathrm{ng} / \mathrm{ml}$ ), por lo que es remitida al Servicio de Medicina Interna para estudio. Cuando la paciente es valorada se encuentra asintomática y la exploración física es rigurosamente normal. Se realiza comprobación de la elevación de CEA $(296.86 \mathrm{ng} / \mathrm{ml})$ y determinación de otros marcadores tumorales (CEA 15.3, CA 19.9, CA 125) los cuales se encuentran en rango normales. El hemograma y la bioquímica también se encuentran dentro de la normalidad. Se procede a descartar patología tumoral digestiva, realizando gastroscopia, en la que se objetiva una gastritis antral (Helicobacter pylori negativa); colonoscopia (hemorroides internas grado II) y ecografía abdominal, que es informada como normal. A continuación se procede a realizar ecografía tiroidea, en la que se objetiva un nódulo único en hemicuerpo tiroideo derecho $(19,6 \times 31,9 \mathrm{~mm})$, bien delimitado y con calcificaciones puntiformes en su interior. Se determina calcitonina $(2.382 \mathrm{pg} / \mathrm{ml}$, rango normal hasta $3.5 \mathrm{pg} / \mathrm{ml}$ ) y cromogranina A (1 nmol/l, normal hasta $4 \mathrm{nmol} / \mathrm{l})$, ante la sospecha que se trata de un carcinoma medular de tiroides (CMT). En la biopsia tiroidea se observó una neoformación sólida

\begin{abstract}
Carcinoembryonic antigen (CEA) is a complex intracellular glycoprotein produced by about $90 \%$ of colorectal cancers and it can be measured quantitatively in serum. It can also be elevated in other conditions such as gastric, pancreatic, lung, breast, medullary thyroid malignancies, as well as in non-neoplastic conditions such as cirrhosis, ulcerative colitis and pancreatitis.
\end{abstract}

Key words: Carcinoembryonic antigen, CEA. Colorectal cancer. Medullary thyroid malignancies.

sin transformación quística, que presenta un crecimiento expansivo periférico y que muestra un contorno neto, parcialmente delimitado por una pseudocápsula de tejido conectivo de espesor uniforme. No se objetiva invasión linfática ni hematógena y no se aprecia diferenciación folicular. La cromatina es fina de distribución homogénea, sin nucleolos prominentes ni hendiduras bien conformadas. Tras la tiroidectomía total se realiza nueva determinación de CEA y calcitonina: 2,48 ng/ml y $15 \mathrm{pg} / \mathrm{mol}$, respectivamente, ambos en rangos normales. Al año de seguimiento la paciente se encuentra asintomática y la analítica es rigurosamente normal (CEA $1.3 \mathrm{ng} / \mathrm{ml})$.

\section{Discusión}

El CEA es una glucoproteína de elevado peso molecular (180 kD) presente en la membrana citoplasmática de numerosas células glandulares y que suele cuantificarse habitualmente en los pacientes que tienen cáncer colorrectal fundamentalmente como diagnóstico precoz de recidiva y como monitorización terapéutica. Se ha observado que su valor aumenta en el postoperatorio de los pacientes con recidivas. Sin embargo, el CEA puede elevarse en otras situaciones, tanto benignas como malignas, además de las neoplasias gastrointestinales (Tabla 1). Se ha observado, además, que el CEA se eleva más en pacientes fumadores.

El CEA no tiene utilidad en el screening del cáncer colorrectal, puesto que carece de especificidad y sensibilidad², especialmente en los estadios más precoces. Por otra parte, algunos autores no han observado que existan diferencias estadísticamente significativas entre los niveles medios del CEA en los pacientes que han presentado un tumor, tanto en el diagnóstico como en la evolución y en los que no lo presentaron $^{3}$. Este hecho resalta el carácter inespecífico de este marcador tumoral, a lo cual hay que añadir el gasto nada desdeñable asociado directa o indirectamente al estudio. 
Tabla 1. Causas de elevación de CEA

Benignas
Hábito tabáquico
Infecciones
Pancreatitis
Enfermedad inflamatoria intestinal
Cirrosis hepática
Tumores benignos del TGl, hígado y páncreas
Neoplasias
Colorrectal
Pancreática
Gástrica
Pulmonar
Mama
Tumores neuroendocrinos, entre ellos carcinoma medular de tiroides

Cerezo et al. han estimado que el gasto medio generado por pacientes en el estudio del despistaje tumorales en relación con la elevación del CEA es de 503,6 $\pm 257,6$ euros $^{3}$.

A pesar de todo en algunos protocolos de salud se ha incorporado su detección como parte de los test de screening en personas asintomáticas. A nuestro juicio en un paciente asintomático con pequeñas elevaciones del CEA $(<20 \mathrm{ng} / \mathrm{ml})$ parece razonable no realizar ningún estudio complementario y simplemente hacer un seguimiento del CEA; mientras que en pacientes asintomáticos con CEA > $200 \mathrm{ng} / \mathrm{ml}$ sí parece oportuno realizar pruebas de despistaje, quedaría por determinar los pacientes en rangos intermedio, en los que habría que individualizar el estudio a realizar. Ante la detección de CEA elevado en un paciente asintomático la mayoría de los protocolos incluyen la realización de endoscopia y pruebas de imagen que incluyan TAC abdomino-pélvico². Si todas las pruebas son negativas el diagnóstico diferencial se debe extender hacia procesos extradigestivos.

El cáncer de tiroides es la neoplasia endocrinológica más frecuente y representa el $1 \%$ de todas las neoplasia malignas. Su incidencia es más frecuente en mujeres (2-4:1). Existen una serie de factores predisponentes, entre los cuales se encuentran los genéticos (mutaciones del prooncogen RET en los carcinomas medulares), radiación externa y exposición a productos químicos (dioxinas, hexaclorobenceno). La forma de presentación más frecuente es como un nódulo tiroideo, aunque también puede debutar como adenopatías cervicales, parálisis del nervio recurrente o metástasis (especialmente en pulmón). En cuanto al CMT, representa el 5-10\% de todas las neoplasias tiroideas y puede aparecer de forma esporádica, Io más habitual, o como una enfermedad familiar autonómica dominante (bien asociada a un síndrome MEN 2A o 2B, o no). EI CMT se puede diseminar por vía hematógena y ganglionar, y la supervivencia a los 5 años es del $30-50 \%{ }^{4}$. Además de la calcitonina el CMT también puede secretar CEA ${ }^{5,6}$, lo cual se asocia a una mayor agresividad tumoral, a un mayor índice de recurrencias y a un peor pronóstico ${ }^{7}$. No existen datos disponibles del porcentaje de pacientes con CMT que tienen CEA elevados. A diferencia de lo que sucede con la calcitonina, Ios niveles de CEA son más estables y no son susceptibles de estimularse tras la administración de calcio o pentagastrina 8 . Así pues, en un paciente con CEA elevado tras descartar patología neoplásica de origen gastrointestinal es obligado descartar patología neuroendocrina, entre la cual se encuentra el carcinoma medular de tiroides 9 .

\section{Bibliografía}

1. Goldstein MJ, Mitchell EP. Carcinoembryonic antigen in the staging and follow-up of patients with colorectal cancer. Cancerlnvest 2005; 23:338-51.

2. Lim Y K, Kam M H, Eu K W. Carcinoembryonic antigen screening: how far should we go? Singapore Med J 2009;50(9): 862.

3. Cerezo A, Rosa F, Lobón JA, Gómez FJ. Capacidad diagnóstica del antígeno carcinoembrionario. Gastroenterol Hepatol. 2014;37(10):551-7.

4. Thompson LD. Medullary thyroid carcinoma. Ear Nose Throat J. 2010;89(7):301-2.

5. Machens A, Dralle H. Biomarker-based risk stratification for previously untreated medullary thyroid cancer. J Clin Endocrinol Metab 2010;95(6):2655-63.

6. Abraham D, Delbridge $L$ et al. Medullary thyroid carcinoma presenting with an initial CEA elevation. ANZ J Surg 2010;80(11):831-3.

7. Meijer JA, le Cessie S et al. Calcitonin and carcinoembryonic antigen doubling times as prognostic factors in medullary thyroid carcinoma: a structured meta-analysis. Clin Endocrinol 2010;72(4):534-42.

8. Osamura RY, Yasuda O, Kawakami T, Itoh Y, Inada K, Kakudo K. Immunoelectron microscopio demonstration of regulated pathway for calcitonin and constitutive pathway for carcinoembryonic antig in the same cells of human medullary arcinomas of thyroid glands. Mod Pathol 1997;10:7-11.

9. Deepak A, Delbridge L, Clifton-Bligh R, Clifton-Bligh P et al. Medullary thyroid carcinoma presenting with an initial CEA elevation. ANZ Journal of Surgery 2010; 80: 831-3. 